AGRICULTURE AND BIOLOGY JOURNAL OF NORTH AMERICA

ISSN Print: 2151-7517, ISSN Online: 2151-7525, doi:10.5251/abjna.2011.2.3.529.537

(C) 2011, ScienceHu $\beta$, http://www.scihub.org/ABJNA

\title{
Weed species composition of citrus -based cropping systems at National Horticultural Research Institute Ibadan, Nigeria.
}

\author{
"Olorunmaiye, P. M ${ }^{1}$., Egberongbe, K.R ${ }^{2}$., Adeoye, P.O' ${ }^{2}$., Alamu, O.O ${ }^{2}$ and Taiwo, S.T \\ ${ }^{1}$ Department of Plant Physiology and Crop Production, University of Agriculture \\ Abeokuta, Abeokuta, Nigeria \\ ${ }^{2}$ National Horticultural Research Institute (NIHORT) Ibadan, Nigeria. \\ *Corresponding author: pmmoji@yahoo.co.uk
}

\begin{abstract}
A preliminary weed survey of citrus orchard and nursery was conducted in National Horticultural Research Institute (NIHORT) Ibadan in the wet season of 2009. The result showed that out of 54 weed species encountered during the survey 39 were broadleaf weeds, 13 were grasses and two were sedges. The main citrus orchard recorded $55 \%$ of the total weed species while the citrus organic orchard recorded the least $(27.8 \%)$. Seven different weed species were propenderant with high Relative Frequencies covering over $5 \%$ of the total weed species in three locations (citrus organic orchard, citrus/vegetable intercrop plot and juvenile citrus plot). Out of these, 3 weed species: Ageratum conyzoides L, Imperata cylindrica L. Raeuschel and Tridax procumbens L. had the greatest Relative Frequency of $12 \%$ each, in citrus organic orchard. However, the rest sampled areas have between 4 and 6 weed species accounting for $5 \%$ or more showing the whole survey area to be very rich in flora. Tridax procumbens $\mathrm{L}$. and Chromolaena odorata (L) R.M Kings and Robinson associated with all the citrus based cropping systems in all the locations. Relative density of Euphorbia heterophylla $L$ (35.95\%) in juvenile citrus orchard was higher than any other species in all the locations. Panicum maximum had the highest Relative Density $(18.8 \%)$ in citrus main orchard among the grass weed species next to Imperata cylindrica L. Raeuschel $(25.09 \%)$ in citrus organic orchard indicating a higher infestation of citrus orchard than other species.
\end{abstract}

Keyword: Citrus, orchard, weed, relative density, relative frequency, organic \section{INTRODUCTION}

Citrus was introduced to Nigeria in the 1930s (Adigun, 1993) and has since spread throughout the country and is currently rated as the most widely planted fruit tree in Nigeria. Citrus currently features in diverse cropping systems which include the multistoried homegardens, cocoa plants, food crop plots and a few pure stand citrus orchard (Amih, 1985, Oladokun, 1990). In NIHORT, citrus is grown as one of the mandate crops and due to its importance, it has received top research attention in the recent time (Aiyelagbe, et al., 2000). At the juvenile stage, citrus is intercropped with cowpea, okra, water melon and maize (NIHORT, 2000) before the canopy closes. The presence of different crop species intercropped with young citrus trees may result in different weed species on such land due to host crop species. In addition, lands under intercropping systems are cropped continuously before the citrus canopy close. Weeds are generally abundant in cultivated land and are subject to changes either in abundance or in weed species present in a locality over a period of time (Yakubu et al., 2006). The National Horticultural Research Institute (NIHORT) is situated in southwestern part of Nigeria where climate favours weed germination and growth almost all the year round. Weeds compete with citrus trees for water, light, space and habour pathogens, rodents and also reduce the efficiency of orchard operations (Futch and Singh, 2008). Some weeds and crops are site specific while others will thrive over a wide range of habitat (Karaye et al., 2007). Recent studies have shown that weed shift occur in continuously cultivated land which may be as a result of high tillage practice, cropping systems, weed control and other changes in the habitat (Smith and Akinde, 2000; Olorunmaiye and Olorunmaiye, 2008). In order to map out weed control strategy for a successful weed control programmes in citrus cropping systems, a good knowledge of the weed type and species composition are necessary in any ecology. Therefore this study was designed to assess weed species composition associated with citrus based cropping systems in NIHORT, in southwest of Nigeria. 


\section{MATERIALS AND METHODS}

The survey was conducted in the wet season of 2009 at the National Horticultural Research Institute (NIHORT), Ibadan (Latitude $7^{\circ} 23^{\prime}$ and $7^{\circ} 25^{\prime} \mathrm{N}$ and longitude $3^{\circ} 50^{\prime}$ and $3^{\circ} 52^{\prime} \mathrm{E}$ ). Six plots (citrus orchard, citrus organic orchard, citrus nursery, citrus weed management plot, citrus /vegetable intercrop plot and citrus juvenile plot) grown to citrus either as a sole crop or intercropped with other crops in different locations within the Institute were surveyed using the quantitative survey method of Thomas 1985. An 'M' pattern was systematically walked in each plot from the edge of each plot and five (5) $0.5 \mathrm{~m}$ $x 0.5 \mathrm{~m}$ quadrats were taken from plots less than 5 hectares, 9 quadrats from $5-15$ hectares and 13 quadrats from over 15 hectares. Weeds within each quadrat were uprooted, sorted into species, identified, counted and recorded. Data collected were subjected to ecological analysis to determine the relative frequency, relative density and summed dominance ratio according to Wirjahadja and Pancho (1975). Only weeds that occurred at $\geq 5 \%$ were considered as being common.

\section{RESULTS}

A total number of 54 weed species belonging to 21 families were encountered in citrus-based cropping systems out of which 39 were broadleaves, 13 were grasses and 2 were sedges (Table 1). Family Poaceae had 14 species, Euphorbiaceae and Asteraceae had 6 each, Amaranthaceae and Fabacea had 3 each, Lamiacea, Malvaceae, Rubiaceae, commelinaceae and urticaceae had 2 each while the remaining families had 1each. Table 2 shows the Relative Frequencies (RF) of the weed species in various locations. In citrus orchard, four species had RF $\geq 5 \%$ with the highest frequency in $I$. triloba $(14.06 \%)$ followed by $A$. gangetica $(9.38 \%)$, while $C$. odorata and $P$. obiculare had $7.81 \%$ each. Three weed species were most frequent in citrus organic plot which are $A$. conyzoides, I. cylindrica and T. procumbens each at $12 \%$ RF. Others with RF $\geq$ $5 \%$ include $C$. pubescens, $C$, odorata, $C$. benghalensis and $P$. maximum at $8 \%$ each. In citrus nursery plot, $T$. procumbens had the highest RF $(11.11 \%)$ while C. odorata, E. heterophylla, E. hirta, I. cylindrica and $M$. villosus recorded $7.41 \%$ RF each. In citrus weed management plot, three weed species: C. benghalensis, $O$. corymbosa and $T$. procumbens each with RF of $10.71 \%$ were most frequent followed by $A$. gangetica, $P$. maximum and $P$. indica at $7.14 \%$ $\mathrm{R} F$ each. In citrus/vegetable plot, $M$. villosus and $T$. procumbens were most frequent (RF $=10.35 \%$ each) while A. sessilis, A. compressus, C.pubescens, C. odorata and S. nodiflora had RF of $6.89 \%$. Centrosema pubescens was the most frequent weed in juvenile citrus plot (RF $=17.86 \%$ ) followed by $E$. heterophylla with RF of $10.71 \%$ while $A$. africana, $A$. gangetica, I. cylindrica P. maximum and S. nodiflora had RF of $7.14 \%$ each.

Table 3 shows the Relative Densities (RD) of the various weed species in citrus-based cropping systems. In citrus orchard, five species had RD $\geq 5 \%$ with the highest in P. maximum (18.83\%) followed by I. cylindrica (11.45\%). Others following in descending order are C. odorata $(6.36 \%)$ A. compressus $(6.11 \%)$ and Perotis indica (5.59\%). In citrus organic orchard, A. conyzoides had the highest RD $(31.92 \%)$ followed by $I$. cylindrica $(21.13 \%)$, T. procumbens $(13.62 \%)$ and $P$. maximum (7.04\%). Ageratum conyzoides and I. cylindrica recorded the highest RD $(23.08 \%$ each) in citrus nursery plot followed by $T$. procumbens, $M$. villosus, $C$. odorata and $B$. arbotiva $(15.00,8.46,8.08$ and $6.15 \%$, respectively) in descending order.

Perotis indica emerged the most abundant weed in citrus weed management plot

$(\mathrm{RD}=29.89 \%)$. This was followed in descending order by $M$. villosus, O. herbacea, $T$. procumbens, $C$. benghalensis and $P$. maximum (11.96, 11.41, 13.04, 9.78 and $6.52 \%$ respectively). In citrus/vegetable plot, $T$. procumbens had the highest RD (16.67\%) followed by $A$. sessilis $(9.52 \%), A$. brasiliana $(6.55 \%)$ and $A$. compressus $(5.36 \%)$. In juvenile citrus plot, $E$. heterophylla recorded the highest abundance with $\mathrm{RD}$ of $35.95 \%, S$. nodiflora, $A$. africana $T$. procumbens, C. pubescens and $P$. indica followed in descending order $(14.64,11.29,7.11,6.28$ and $6.28 \%$ respectively).

Table 4 shows the summed dominant ratio of the weed species in citrus-based cropping system. Five weed species were dominant in citrus orchard plot with $P$. maximum (13.32) being most dominant. Others in descending are I. triloba (9.07), C. odorata (7.09), A. gangetica (6.73) and A. gayanus (5.67). Ageratum conyzoides was the most dominant weed in citrus organic orchard followed in descending order by $I$. cylindrica (16.57), T. procumbens (12.81), $P$. maximum (7.52) and $C$. benghalensis (5.88). In citrus nursery plot $l$. cylindrica (15.25) was the most dominant weed followed by $A$. conyzoides (13.39), $M$. villosus (7.94) and C. odorata (7.76). Six weeds were dominant in citrus weed management plot with $P$. indica being the most dominant (18.52). Others include $T$. procumbens (11.87), O. corymbosa 
Agric. Biol. J. N. Am., 2011, 2(3): 529-537

(11.06), C. benghalensis (10.24), M. villosus (7.77) and $P$. maximum (6.83). In citrus/vegetable plot, five weeds were dominant with $T$. procumbens most dominant (13.51) while $A$. sessilis (8.21), M. villosus (7.26), A. compressus (6.13) and C. pubescens
(5.23) followed in descending order. Juvenile Citrus plot recorded six weed species as being dominant with $E$. heterophylla being most dominant (23.35) followed by C. pubescens (12.07) and T. procumbens (5.34). 
Table 1: Weed species encountered in all the citrus based plots inNIHORT, Ibadan, Nigeria in 2009.

\begin{tabular}{|c|c|c|}
\hline Weed species & Family & Growth form \\
\hline Adenopus breviflorus Benth G. Roberty & Cucubitaceae & $\mathrm{ABL}$ \\
\hline Alchornea laxiflora(Benth.) Pax \& K Hoffin & Euphobiaceae & PBL \\
\hline Alternanthera brasiliana & Amaranthaceae & $\mathrm{ABL}$ \\
\hline Alternanthera sessilis (L.) R. Br. ex Roth & Amaranthaceae & $A B L$ \\
\hline Andropogon gayanus Kunth & Poaceae & AG \\
\hline Ageratum conyzoides $\mathrm{L}$. & Asteraceae & $\mathrm{ABL}$ \\
\hline Aspilia africana (Pers.)c. Adams & Asteraceae & $\mathrm{ABL}$ \\
\hline Aspilia latifolia Oliv. \& Hiern & Asteraceae & $\mathrm{ABL}$ \\
\hline Asystasia gangetica (Linn.)T. Anders. & Acanthaceae & $\overline{A B L}$ \\
\hline Axonopus compressus (Sw.) P. Beauv. & Poaceae & $A G$ \\
\hline Boerhavia diffusa $\mathrm{L}$. & Nyctaginaceae & $\mathrm{ABL}$ \\
\hline Bulbostylis arbotiva (Steudel) & Poaceae & $A G$ \\
\hline Calopogonium mucunoides Desv & Fabaceae & $\mathrm{ABL}$ \\
\hline Centrosema pubescens Benth. & Fabaceae & PBL \\
\hline Chamaecrista mimosoides (L.) Greene & Mimosoidae & $\mathrm{ABL}$ \\
\hline Chromolaena odorata (L.) R.M.Kings \& Robinson & Asteraceae & PBL \\
\hline Corchorus olitorius L. & Tiliaceae & $\mathrm{ABL}$ \\
\hline Commelina benghalensis L. & Commelinaceae & $\mathrm{ABL}$ \\
\hline Commelina erecta $L$. & Commelinaceae & $\mathrm{ABL}$ \\
\hline Croton sp & Euphobiaceae & $\overline{A B L}$ \\
\hline Cyperus esculentus Linn. & Cyperaceae & PS \\
\hline Digitaria horizontalis Willd. & Poaceae & AG \\
\hline Desmodium scorpiurus (Sw.) Desv. & Fabaceae & $\mathrm{ABL}$ \\
\hline Eleusine indica Gaertn. & Poaceae & $A G$ \\
\hline Eragrostis tenella (Linn.) P. Beauv. Ex Roem \& Schult. & Poaceae & AG \\
\hline Euphorbia heterophylla Linn. & Euphobiaceae & $\mathrm{ABL}$ \\
\hline Euphorbia hirta Linn. & Euphobiaceae & $A B L$ \\
\hline Euphorbia hysopifollia Linn. & Euphobiaceae & $\mathrm{ABL}$ \\
\hline Ficus exasperata Vahl & Moraceae & PBL \\
\hline Gomphrena celosoides Mart. & Amaranthaceae & $\mathrm{ABL}$ \\
\hline Imperata cylindrica L. Raeuschel & Poaceae & $P G$ \\
\hline Ipomoea triloba Linn. & Convulvulacea & $\mathrm{ABL}$ \\
\hline Laportea aetuans (Linn.) Chew. & Urticaceae & $\mathrm{ABL}$ \\
\hline Leucaena leucocephalla & Fabaceae & PBL \\
\hline Mimosa pudica Linn. & Mimosoideae & $\mathrm{ABL}$ \\
\hline Mitracarpus villosus (SW) DC & Rubiaceae & $\mathrm{ABL}$ \\
\hline Oldenlandia herbacea (Linn.) Roxb. & Rubiaceae & $\mathrm{ABL}$ \\
\hline Panicum maximum Jacq. O. Ktze & Poaceae & PG \\
\hline Paspalum obiculare Forst. & Poaceae & $A G$ \\
\hline Pennisetum pedicellatum Trin. & Poaceae & $A G$ \\
\hline Perotis indica Linn. & Poaceae & $A G$ \\
\hline Phyllanthus amarus Schum, et Thonn. & Euphorbiaceae & $\mathrm{ABL}$ \\
\hline Platostoma africanum P.Beauv. & Lamiaceae & $\mathrm{ABL}$ \\
\hline Pouzolzia guineensis Benth. & Urticaceae & $\mathrm{ABL}$ \\
\hline Setaria barbata (Lam.) Kunth. & Poaceae & $A G$ \\
\hline Sida acuta Burm.f. & Malvaceae & $\mathrm{ABL}$ \\
\hline Sida cordiflora Linn. & Malvaceae & $\mathrm{ABL}$ \\
\hline Spigelia anthelmia Linn. & Loganiaceae & $\mathrm{ABL}$ \\
\hline Solenostemon monostachyus (P.Beauv.)Brig. & Lamiaceae & $\mathrm{ABL}$ \\
\hline Sporobolus pyramidalis P.Beauv. & Poaceae & $A G$ \\
\hline Sorghum $s p$ & Poaceae & $A G$ \\
\hline Synedrella nodiflora Gaertn. & Asteraceae & $\mathrm{ABL}$ \\
\hline Talinum triangulare (Jacq.) Willd. & Portulacaceae & $\mathrm{ABL}$ \\
\hline Tridax procumbens L. & Asteraceae & $\mathrm{ABL}$ \\
\hline
\end{tabular}

ABL : Annual Broadleaf; AG: Annual Grass; PG: Perennial Grass; PBL: Perennial Broadleaf; PS: Perennial Sedge 
Table 2: Relative Frequencies of weed species composition in citrus based cropping systems in NIHORT, Ibadan, Nigeria.

\begin{tabular}{|c|c|c|c|c|c|c|}
\hline Weeds species & $\begin{array}{l}\text { Citrus } \\
\text { orchard }\end{array}$ & $\begin{array}{l}\text { Citrus } \\
\text { organic }\end{array}$ & $\begin{array}{l}\text { Citrus } \\
\text { nursery }\end{array}$ & $\begin{array}{l}\text { Citrus } \\
\text { wmgt }\end{array}$ & Citrus/veg & $\begin{array}{l}\text { Juvenile } \\
\text { citrus }\end{array}$ \\
\hline Adenopus breviflorus & - & - & - & 3.57 & - & - \\
\hline $\begin{array}{l}\text { Alchornea laxiflora (Benth.) Pax \& } \\
\text { K Hoffin }\end{array}$ & 1.56 & - & - & - & - & - \\
\hline Alternanthera brasiliana & 1.56 & 4 & - & - & 3.45 & - \\
\hline $\begin{array}{l}\text { Alternanthera sessilis (L.) R. Br. } \\
\text { ex Roth }\end{array}$ & - & - & - & - & 6.89 & - \\
\hline Andropogon gayanus Kunth & 3.13 & - & - & - & - & - \\
\hline Ageratum conyzoides & - & 12 & 3.7 & 3.57 & 3.45 & - \\
\hline Aspilia africana (Pers.)c. Adams & 3.13 & 4 & - & 3.57 & - & 7.14 \\
\hline Aspilia latifolia & 1.56 & - & - & - & - & - \\
\hline $\begin{array}{l}\text { Asystasia gangetica (Linn.)T. } \\
\text { Anders. }\end{array}$ & 9.38 & - & 3.7 & 7.14 & 3.45 & 7.14 \\
\hline $\begin{array}{l}\text { Axonopus compressus (Sw.) P. } \\
\text { Beauv. }\end{array}$ & 3.13 & 4 & - & - & 6.89 & 3.57 \\
\hline Boerhavia diffusa L. & - & - & 3.7 & - & - & - \\
\hline Bulbostylis arbotiva (Steudel) & - & - & 3.7 & - & - & - \\
\hline Calopogonium mucunoides Desv & 4.69 & - & 3.7 & - & 3.45 & 3.57 \\
\hline Centrosema pubescens Benth. & 4.69 & 8 & 3.7 & - & 6.89 & 17.86 \\
\hline $\begin{array}{l}\text { Chamaecrista mimosoides (L.) } \\
\text { Greene }\end{array}$ & 1.56 & - & - & - & - & - \\
\hline $\begin{array}{l}\text { Chromolaena odorata (L.) } \\
\text { R.M.Kings \& Robinson }\end{array}$ & 7.81 & 8 & 7.41 & 3.57 & 6.89 & 3.57 \\
\hline Corchorus olitorius & 1.56 & - & - & - & - & - \\
\hline Commelina benghalensis L. & 3.13 & 8 & - & 10.7 & - & - \\
\hline Commelina erecta $L$ & - & - & - & - & - & 3.57 \\
\hline Croton sp & 1.56 & - & - & - & - & - \\
\hline Cyperus esculentus Linn. & - & - & - & 3.57 & 3.45 & - \\
\hline Digitaria horizontalis Willd. & 1.56 & - & - & - & - & - \\
\hline $\begin{array}{l}\text { Desmodium scorpiurus (Sw.) } \\
\text { Desv. }\end{array}$ & - & 4 & 3.7 & - & 3.45 & - \\
\hline Eleusine indica Gaertn. & 1.56 & - & - & - & - & - \\
\hline $\begin{array}{l}\text { Eragrostis tenella (Linn.) P. } \\
\text { Beauv. Ex Roem \& Schult. }\end{array}$ & - & - & - & - & 3.45 & 10.71 \\
\hline Euphorbia heterophylla Linn. & - & - & 7.41 & - & 3.45 & - \\
\hline Euphorbia hirta Linn. & - & - & 7.41 & - & - & - \\
\hline Euphorbia hysopifollia Linn. & - & - & 3.7 & - & - & - \\
\hline Ficus exasperata Vahl & - & - & - & - & 3.45 & - \\
\hline Gomphrena celosoides Mart. & 1.56 & - & 3.7 & - & - & - \\
\hline Imperata cylindrica L. Raeuschel & 4.69 & 12 & 7.41 & 3.57 & 3.45 & - \\
\hline Ipomoea triloba Linn. & 14.06 & 4 & - & 3.57 & 3.45 & 7.14 \\
\hline Laportea aetuans (Linn.) Chew. & 1.56 & - & - & 3.57 & - & - \\
\hline Leucaena leucocephalla & - & - & - & 3.57 & - & - \\
\hline Mimosa pudica Linn. & - & - & - & - & 3.45 & - \\
\hline Mitracarpus villosus (SW) DC & 1.56 & - & 7.41 & 3.57 & 10.35 & - \\
\hline $\begin{array}{l}\text { Oldenlandia herbacea (Linn.) } \\
\text { Roxb. }\end{array}$ & - & - & 3.7 & 10.71 & - & - \\
\hline Panicum maximum Jacq. O. Ktze & 7.81 & 8 & - & 7.14 & - & 7.14 \\
\hline Paspalum obiculare Forst. & - & 4 & 3.7 & - & 3.45 & - \\
\hline Pennisetum pedicellatum Trin. & - & 4 & - & - & - & - \\
\hline Perotis indica Linn. & 1.56 & - & - & 7.14 & - & 3.57 \\
\hline $\begin{array}{l}\text { Phyllanthus amarus Schum, et } \\
\text { Thonn. }\end{array}$ & - & - & 3.7 & - & - & - \\
\hline
\end{tabular}




\begin{tabular}{|l|l|l|l|l|l|l|}
\hline Platostoma africanum P.Beauv. & - & - & - & 3.57 & - & - \\
\hline Pouzolzia guineensis Benth. & - & - & - & 3.57 & - & - \\
\hline Setaria barbata (Lam.) Kunth. & 1.56 & - & - & - & - & - \\
\hline Sida acuta Burm.f. & 3.13 & - & - & - & - & - \\
\hline Sida cordiflora Linn. & 3.13 & - & - & - & - & 3.57 \\
\hline Spigelia anthelmia Linn. & - & - & 3.7 & - & - & 3.57 \\
\hline $\begin{array}{l}\text { Solenostemon monostachyus } \\
\text { (P.Beauv.)Brig. }\end{array}$ & - & - & 3.57 & - & - & - \\
\hline Sporobolus pyramidalis P.Beauv. & 1.56 & - & - & - & - & - \\
\hline Sorghum sp & - & - & - & - & - & 3.57 \\
\hline Synedrella nodiflora Gaertn. & 1.56 & - & - & 3.57 & 6.89 & 7.14 \\
\hline Talinum triangulare (Jacq.) Willd. & 3.13 & 4 & - & - & - & 3.57 \\
\hline Tridax procumbens L. & 4.69 & 12 & 11.11 & 10.71 & 10.35 & 3.57 \\
\hline
\end{tabular}

Citrus/veg = Citrus/vegetable plot

Citrus/wmgt $=$ Citrus/weed management plot

Table 3: Weed Relative Densities (\%) of different weed species in citrus-based cropping systems in NIHORT, Ibadan, Nigeria.

\begin{tabular}{|c|c|c|c|c|c|c|}
\hline Weeds species & $\begin{array}{l}\text { Citrus } \\
\text { orchard }\end{array}$ & $\begin{array}{l}\text { Citrus } \\
\text { organic }\end{array}$ & $\begin{array}{l}\text { Citrus } \\
\text { nursery }\end{array}$ & $\begin{array}{l}\text { Citrus } \\
\text { wmgt }\end{array}$ & Citrus/veg & $\begin{array}{l}\text { Juvenile } \\
\text { citrus }\end{array}$ \\
\hline Adenopus breviflorus & - & - & - & 0.54 & - & - \\
\hline $\begin{array}{l}\text { Alchornea laxiflora (Benth.) Pax \& } \\
\text { K Hoffin }\end{array}$ & 0.25 & - & - & - & - & - \\
\hline Alternanthera brasiliana & 0.25 & 0.9 & - & - & 6.55 & - \\
\hline $\begin{array}{l}\text { Alternanthera sessilis (L.) R. Br. } \\
\text { ex Roth }\end{array}$ & - & - & - & - & 9.52 & - \\
\hline Andropogon gayanus Kunth & 4.83 & - & - & - & - & - \\
\hline Ageratum conyzoides $\mathrm{L}$. & - & 31.9 & 23.8 & 2.72 & 0.59 & - \\
\hline Aspilia africana (Pers.)c. Adams & 2.54 & 3.8 & - & 1.08 & - & 11.29 \\
\hline Aspilia latifolia & 3.05 & - & - & - & - & - \\
\hline $\begin{array}{l}\text { Asystasia gangetica (Linn.)T. } \\
\text { Anders. }\end{array}$ & 4.07 & - & 0.39 & 1.08 & 0.59 & 4.6 \\
\hline $\begin{array}{l}\text { Axonopus compressus (Sw.) P. } \\
\text { Beauv. }\end{array}$ & 6.11 & 4.6 & - & - & 5.36 & 3.35 \\
\hline Boerhavia diffusa L. & - & - & 0.39 & - & - & - \\
\hline Bulbostylis arbotiva (Steudel) & - & - & 6.15 & - & - & - \\
\hline Calopogonium mucunoides Desv & 1.02 & - & 0.39 & - & 0.59 & 1.26 \\
\hline Centrosema pubescens Benth. & 1.53 & 1.88 & 0.39 & - & 3.57 & 28 \\
\hline $\begin{array}{l}\text { Chamaecrista mimosoides (L.) } \\
\text { Greene }\end{array}$ & 0.25 & - & - & - & - & - \\
\hline $\begin{array}{l}\text { Chromolaena odorata (L.) } \\
\text { R.M.Kings \& Robinson }\end{array}$ & 6.36 & 0.94 & 8.08 & 0.54 & 2.98 & 0.42 \\
\hline Corchorus olitorius & 0.25 & - & - & - & - & - \\
\hline Commelina benghalensis L. & 4.58 & 3.76 & - & 9.78 & - & - \\
\hline Commelina erecta $L$. & - & - & - & - & - & 2.09 \\
\hline Croton $s p$ & 0.76 & - & - & - & - & - \\
\hline Cyperus esculentus Linn. & - & - & - & 2.17 & 0.59 & - \\
\hline Digitaria horizontalis Willd. & 0.25 & - & - & - & - & - \\
\hline $\begin{array}{l}\text { Desmodium scorpiurus (Sw.) } \\
\text { Desv. }\end{array}$ & - & 2.35 & 0.39 & - & 0.59 & - \\
\hline Eleusine indica Gaertn. & 0.73 & - & - & - & - & - \\
\hline $\begin{array}{l}\text { Eragrostis tenella (Linn.) P. } \\
\text { Beauv. Ex Roem \& Schult. }\end{array}$ & - & - & - & - & 1.79 & - \\
\hline Euphorbia heterophylla Linn. & - & - & 1.92 & - & 3.57 & 35.98 \\
\hline Euphorbia hirta Linn. & - & - & 1.15 & - & - & - \\
\hline Euphorbia hysopifollia Linn. & - & - & 0.39 & - & - & - \\
\hline Ficus exasperata Vahl & - & - & - & - & 3.45 & - \\
\hline
\end{tabular}




\begin{tabular}{|l|l|l|l|l|l|l|}
\hline Gomphrena celosoides Mart. & 4.07 & - & 2.31 & - & - & - \\
\hline Imperata cylindrica L. Raeuschel & 11.45 & 21.13 & 23.08 & 2.17 & 1.79 & - \\
\hline Ipomoea triloba Linn. & 4.07 & 0.46 & - & 1.07 & 0.59 & 1.68 \\
\hline Laportea aetuans (Linn.) Chew. & 0.25 & - & - & 3.26 & - & - \\
\hline Leucaena leucocephalla & - & - & - & 0.54 & - & - \\
\hline Mimosa pudica Linn. & - & - & - & - & 0.59 & - \\
\hline Mitracarpus villosus (SW) DC & 2.29 & - & 8.46 & 11.96 & 4.17 & - \\
\hline $\begin{array}{l}\text { Oldenlandia herbacea (Linn.) } \\
\text { Roxb. }\end{array}$ & - & 2.04 & 3.08 & 11.41 & - & - \\
\hline Panicum maximum Jacq. O. Ktze & 18.83 & 7.04 & - & 6.52 & - & 1.26 \\
\hline Paspalum obiculare Forst. & - & 0.94 & 1.15 & - & 0.59 & - \\
\hline Pennisetum pedicellatum Trin. & - & 0.94 & - & - & - & - \\
\hline Perotis indica Linn. & 5.59 & 0.94 & - & 29.89 & - & 6.28 \\
\hline Phyllanthus amarus Schum, et \\
Thonn. & - & - & 0.77 & - & - & - \\
\hline Platostoma africanum P.Beauv. & - & - & - & 0.54 & - & - \\
\hline Pouzolzia guineensis Benth. & - & - & - & 0.54 & - & - \\
\hline Setaria barbata (Lam.) Kunth. & 3.56 & - & - & - & - & - \\
\hline Sida acuta Burm.f. & 1.27 & - & - & - & - & - \\
\hline Sida cordiflora Linn. & 3.05 & - & - & - & - & 0.42 \\
\hline Spigelia anthelmia Linn. & - & - & 2.69 & - & - & 1.67 \\
\hline $\begin{array}{l}\text { Solenostemon monostachyus } \\
\text { (P.Beauv.)Brig. }\end{array}$ & - & - & 2.69 & - & - & - \\
\hline Sporobolus pyramidalis P.Beauv. & 1.27 & - & - & - & - & - \\
\hline Sorghum sp & - & - & - & - & - & 1.26 \\
\hline Synedrella nodiflora Gaertn. & 0.51 & - & - & 0.54 & 2.38 & 14.64 \\
\hline Talinum triangulare (Jacq.) Willd. & 1.01 & 4.69 & - & - & - & 0.42 \\
\hline Tridax procumbens L. & 4.33 & 13.62 & 15 & 13.04 & 16.67 & 7.11 \\
\hline Cirus & & & & & \\
\hline
\end{tabular}

Citrus/veg $=$ Citrus/vegetable plot

Citrus/wmgt $=$ Citrus/weed management plot

Table 4: Summed dominance ratio of the weed species in citrus based cropping systems In NIHORT, Ibadan, Nigeria.

\begin{tabular}{|l|l|l|l|l|l|l|l|}
\hline Weeds species & & $\begin{array}{l}\text { Citrus } \\
\text { orchard }\end{array}$ & $\begin{array}{l}\text { Citrus } \\
\text { organic }\end{array}$ & $\begin{array}{l}\text { Citrus } \\
\text { nursery }\end{array}$ & $\begin{array}{l}\text { Citrus } \\
\text { wmgt }\end{array}$ & Citrus/veg & $\begin{array}{l}\text { Juvenile } \\
\text { citrus }\end{array}$ \\
\hline Adenopus breviflorus & & - & - & - & 2.06 & - & - \\
\hline $\begin{array}{l}\text { Alchornea laxiflora (Benth.) Pax \& K } \\
\text { Hoffin }\end{array}$ & & - & 0.91 & - & - & - & - \\
\hline Alternanthera brasiliana & & - & 0.91 & 2.5 & - & - & 2 \\
\hline Alternanthera sessilis (L.) R. Br. ex Roth & & - & - & - & - & 8.21 & - \\
\hline Andropogon gayanus Kunth & & 5.67 & - & - & - & - & - \\
\hline Ageratum conyzoides & & - & 21.9 & 13.39 & 3.15 & 2.02 & - \\
\hline Aspilia Africana (Pers.)c. Adams & & 2.84 & 3.9 & - & 2.33 & - & 9.22 \\
\hline Aspilia latifolia & & 2.31 & - & - & - & - & - \\
\hline Asystasia gangetica (Linn.)T. Anders. & & 6.73 & - & 2.05 & 4.11 & 2.02 & 5.67 \\
\hline Axonopus compressus (Sw.) P. Beauv. & & 4.62 & 4.3 & - & - & 6.13 & 3.46 \\
\hline Boerhavia diffusa L. & & - & - & 2.05 & - & - & - \\
\hline Bulbostylis arbotiva (Steudel) & & - & - & 4.93 & - & - & - \\
\hline Calopogonium mucunoides Desv & & 2.83 & - & 2.05 & - & 2.02 & 2.42 \\
\hline Centrosema pubescens Benth. & & 3.11 & 4.9 & 2.05 & - & 5.23 & 12.07 \\
\hline Chamaecrista mimosoides (L.) Greene & & 0.91 & - & - & - & - & - \\
\hline $\begin{array}{l}\text { Chromolaena odorata (L.) R.M.Kings \& } \\
\text { Robinson }\end{array}$ & 7.09 & 4.5 & 7.75 & 2.06 & 4.94 & 1.99 \\
\hline
\end{tabular}




\begin{tabular}{|c|c|c|c|c|c|c|}
\hline Corchorus olitorius & 0.91 & - & - & - & - & - \\
\hline Commelina benghalensis L. & 3.86 & 5.88 & - & 10.24 & - & - \\
\hline Commelina erecta $L$. & - & - & - & - & - & 2.83 \\
\hline Croton sp & 1.16 & - & - & - & - & - \\
\hline Cyperus esculentus Linn. & - & - & - & 2.87 & 2.07 & - \\
\hline Digitaria horizontalis Willd. & 0.91 & - & - & - & - & - \\
\hline Desmodium scorpiurus (Sw.) Desv. & - & 3.2 & 2.05 & - & 2.07 & - \\
\hline Eleusine indica Gaertn. & 1.15 & - & - & - & - & - \\
\hline $\begin{array}{l}\text { Eragrostis tenella (Linn.) P. Beauv. Ex } \\
\text { Roem \& Schult. }\end{array}$ & - & - & - & - & - & 2.62 \\
\hline Euphorbia heterophylla Linn. & - & - & 4.67 & - & 3.51 & 23.35 \\
\hline Euphorbia hirta Linn. & - & - & 4.28 & - & - & - \\
\hline Euphorbia hysopifollia Linn. & - & - & 2.05 & - & - & - \\
\hline Ficus exasperata Vahl & - & - & - & - & 2.32 & - \\
\hline Gomphrena celosoides Mart. & 2.82 & - & 3.01 & - & - & - \\
\hline Imperata cylindrica L. Raeuschel & 0.07 & 16.5 & 15.25 & 2.87 & 2.62 & - \\
\hline Ipomoea triloba Linn. & 9.07 & 2.2 & - & 2.32 & 2.02 & 4.41 \\
\hline Laportea aetuans (Linn.) Chew. & 0.91 & - & - & 3.42 & - & - \\
\hline Leucaena leucocephalla & - & - & - & 2.06 & - & - \\
\hline Mimosa pudica Linn. & - & - & - & - & 2.02 & - \\
\hline Mitracarpus villosus (SW) DC & 1.93 & - & 7.94 & 7.77 & 7.26 & - \\
\hline Oldenlandia herbacea (Linn.) Roxb. & - & - & 3.39 & 11.06 & - & - \\
\hline Panicum maximum Jacq. O. Ktze & 13.32 & 7.52 & - & 6.83 & - & 4.2 \\
\hline Paspalum obiculare Forst. & - & 2.5 & 2.43 & - & 2.02 & - \\
\hline Pennisetum pedicellatum Trin. & - & 2.5 & - & - & - & - \\
\hline Perotis indica Linn. & 3.58 & - & - & 18.52 & - & 4.93 \\
\hline Phyllanthus amarus Schum, et Thonn. & - & - & 2.24 & - & - & - \\
\hline Platostoma africanum P.Beauv. & - & - & - & 2.06 & - & - \\
\hline Pouzolzia guineensis Benth. & - & - & - & 2.06 & - & - \\
\hline \multicolumn{7}{|l|}{ Setaria barbata (Lam.) Kunth. } \\
\hline Sida acuta Burm.f. & 2.2 & - & - & - & - & - \\
\hline Sida cordiflora Linn. & 3.09 & - & - & - & - & 1.99 \\
\hline Spigelia anthelmia Linn. & - & - & 3.2 & - & - & 2.62 \\
\hline $\begin{array}{l}\text { Solenostemon monostachyus } \\
\text { (P.Beauv.)Brig. }\end{array}$ & - & - & 3.2 & - & - & - \\
\hline Sporobolus pyramidalis P.Beauv. & 1.42 & - & - & - & - & - \\
\hline Sorghum sp & - & - & - & - & - & 2.42 \\
\hline Synedrella nodiflora Gaertn. & 1.04 & - & - & 2.06 & 4.64 & 10.89 \\
\hline Talinum triangulare (Jacq.) Willd. & 2.07 & 4.4 & - & - & - & 1.99 \\
\hline Tridax procumbens $\mathrm{L}$. & 4.51 & 12.8 & 13.06 & 11.87 & 13.51 & 5.34 \\
\hline
\end{tabular}

Citrus/veg = Citrus/vegetable plot

Citrus $/$ wmgt $=$ Citrus $/$ weed management plot

\section{DISCUSSION}

Broadleaf weeds generally dominated all the surveyed locations than grasses and sedges and higher concentration of the broadleaf weeds was observed under the canopies of mature citrus trees than the alleys. This may be a preference of broadleaf weeds for shade than full and uninterrupted light intensity in the alleys. The citrus orchard recorded the highest number of weed species (30 species) followed by citrus/vegetable intercrop plot (20 species) with the least in citrus organic plot (15 species). The higher number of weed species observed in the main citrus orchard might be due to the fact that the orchard is the oldest and probably has attained a stable biodiversity. This confirms the earlier report of Yakubu et al., (2006) who reported site specificity in both crops and weeds. Tridax procumbens $\mathrm{L}$. and $C$. odorata associated with all the citrus cropping systems in all the locations and both recorded Relative frequencies greater than $5 \%$ in four of the six locations surveyed. The two species belong to the family Asteraceae with high fruit production and efficient fruit and seed dispersal mechanisms readily brought about by wind, coupled with this is 
high colonizing power of this family. Three of the locations: citrus organic orchard, citrus/vegetable intercrop plots and juvenile citrus plot had 7 weed species to be preponderant covering over $5 \%$ of the total weed species populations sampled while the rest sampled areas have between four and six weed species accounting for $5 \%$ or more showing the whole surveyed areas to be very rich in flora diversities which indirectly is an indication of adequate availability of nutrients in these locations. Euphorbia heterophylla L. recorded the highest relative weed density (35.98\%) in juvenile citrus plot compared to the other weed species in other locations. This may be attributed to its high light requirement, aggressive growth, short life cycle, large seed production with potent explosive seed dispersal mechanism. Panicum maximum Jacq.O. Ktze had the highest relative weed density $(18.8 \%)$ in citrus main orchard among grass weed species next to $I$. cylindrica $(25.09 \%)$ in citrus organic orchard. This is an indication of higher infestation of citrus orchard than other species.

\section{REFERENCES}

Adigun, J.A.; S.T.O. Lagoke; V. Kurmar and I.D. Erinle, (1993). Weed management studies in transplanted tomato in the Nigreian savanna. Samaru Journal of Agricultural Research 10: 29-39.

Aiyelagbe, I.O.O.; S.O. Afolayan.; O.O. Odeleye; L.O. Ogungbayigbe and A.O. Olufoalji (2001). Citrus production in the savanna of Western Nigeria: Current status and opportunities for research input in: book of abstracts and proceedings. Deutshe Tropentag 2001 Conference on International Agricultural Research 4 DEV 9-10 Oct. Univ. Bonn. Germany pp33.

Amih, C.A. (1985). Citrus production in Nigeria. In : Proceedings of National Fruits Workshop. Federal Agricultural Coordinating Unit (FACU) Sponsor Ibadan, Nigeria pp 42-49.

Frick, B. A and A.G. Thomas (1992). Weed surveys in different tillage systems in Southwestern Ontario field crops. Canadian Journal of Plant Science 72: pp13371347.

Futch, S.H. and M. Singh (2007). Weeds in Florida Citrus New Challenges and Opportunities. Citrus Industry Magazine 88(3): 15-17.

Karaye A.K.; A.I. Yakubu and M. Aliyu (2007). Checklist of weeds in irrigated garlic (Allium sativum) and onion (Allium cepa) in Sokoto River Valley. Nigreian Journalof Weed Science volume 20, pp53-57.

National Horticultural Research Institute (2000). 25 years research into horticultural crops development in Nigeria (1975-2000); Denton, O.A.; K.O Alasiri and M.A.Adejoro (eds.). $25^{\text {th }}$ Anniversary Commemorative Book. 140pp.

Obadoni, B.O. and S.U. Remison (2004). The weed flora of upland rice farms in Edo state, Nigerian Journal of Weed Science vol 17 pp1-8.

Oladokun, M.O.A. (1990). Treecrop based agroforestry in Nigeria. A checklist list of some crops intercropped with cocoa. Agroforestry System 5: 153-168.

Olorunmaiye, K. S and Olorunmaiye, P. M. (2008). Influence of weed control methods on weed flora of a Cowpea plot in an ecological zone of southern Guinea savanna of Nigeria. Biological and Environmental Sciences Journal for the Tropics (BEST) 5 (2): 146-150 Published by the Department of Biological Sciences .Faculty of Science, Bayero University, Kano Nigeria.

Smith, M.A. and A.A. Akinde (2000). Comparative weed succession in uncropped and cropped land in a tropical rain forest zone. African Journal of Environmental Studies vol.1 (1) pp104-114.

Thomas, A.G. 1985). Weed survey systems used in Saskatcheuan for cereal and oilseed crops. Weed Science 33: 34-43.

Yakubu, A.I.; J. Alhassan; A. Lado and Sarkindiya (2006). Comparative weed density studies in irrigated carrot (Daucus carota L.), potato (Solanum tuberosus) and wheat (Triticum aestivum Linn.) in Sokoto Rima Valley. Journal of Plant Science 1 (1) : USA. pp14-21. 